\title{
Unplanned Disruption Analysis in Urban Railway Systems Using Smart Card Data
}

\author{
Tianyou Liu ${ }^{1} \cdot{\text { Zhenliang } \mathrm{Ma}^{2} \cdot \text { Haris N. Koutsopoulos }}^{1}$
}

Received: 23 February 2021/Revised: 3 April 2021/Accepted: 24 April 2021 / Published online: 1 June 2021

(C) The Author(s) 2021

\begin{abstract}
Metro system disruptions are a big concern due to their impacts on safety, service quality, and operating efficiency. A better understanding of system performance and passenger behavior under unplanned disruptions is critical for efficient decision making, effective customer communication, and identifying potential improvements. However, few studies explore disruption impacts on individual passenger behavior, and most studies use manually collected survey data. This study examines the potential of using automated collection data to comprehensively analyze unplanned disruption impacts. We propose a systematic approach to evaluate disruption impacts on system performance and individual responses in urban railway systems using automated fare collection (AFC) data. We develop a set of performance metrics to evaluate performance from the perspectives of train operations, information provision (communication), and bridging strategy (shuttle bus services to connect stations impacted by a disruption). We also propose an inference method to quantify the individual response to disruptions (e.g. travel or not, change stations or modes) depending on their trip characteristics with respect to the location and timing of the disruption. The proposed approach is demonstrated using data from a busy metro system. The results highlight the ability of AFC data in providing new insights for the
\end{abstract}

Zhenliang Ma

mike.ma@monash.edu

1 Department of Civil and Environmental Engineering, Northeastern University, Boston, MA 02115, United States

2 Institute of Transport Studies, Department of Civil Engineering, Monash University, Clayton, Victoria 3800, Australia

Communicated by Xuesong Zhou. analysis of unplanned disruptions, which are difficult to extract from traditional data collection methods. The case study shows that the disruption impacts are network-wide, and the impacts on passengers continue for a significant amount of time after the incident ended. The behavior highlights the importance of real-time information and the need for timely dissemination.

Keywords Metro unplanned disruptions - Automated Fare Collection data $\cdot$ System performance $\cdot$ Passenger response $\cdot$ Bus bridging

\section{Introduction}

Given the fast-increasing ridership in big cities, metro systems have become more vulnerable when incidents occur [1]. Disruptions can lead to degradation of normal service levels, resulting in significant impacts on operations and passengers. Therefore, it is critical to understand how the system performs and how passengers respond to disruptions in order to develop effective management strategies to mitigate their impacts.

Disruptions can be planned or unplanned. This paper focuses on unplanned disruptions, such as train signaling failure, operation errors, and human accidents. Unplanned disruptions can differ in magnitude and location. Severe disruptions, e.g. signal failures, may result in partial or entire system shutdown. Since unplanned disruptions are unpredictable, passenger response largely depends on operating management strategies. Information provision is especially important in helping passengers plan/adjust their travel during disruptions.

Several studies have explored the disruption impacts on system performance using analytical and simulation 
models or incident logs. For example, Jenelius et al. [2] developed a model to assess rail transit robustness and redundancy during planned incidents (line extension). The model quantifies the passenger welfare by considering passengers' dynamic travel choices, traffic conditions and other factors. They evaluated various disruption scenarios considering user behavior, traffic, and train capacities. Adnan et al. [3] developed a multi-modal simulation tool to evaluate the metro system performance. The tool is based on hierarchical discrete choice models using a monte-carlo simulation approach, and it can assess impacts on disruptions and test various mitigation strategies. They evaluated the metro system performance under a major unplanned incident from perspectives of average journey time, dwell time, waiting time, number of denied boarding, and mode share. Yap et al. [4] proposed a supervised learning model to predict the number of disruptions by incident type, location, and time of incidents in the Washington D.C. metro system. Yin et al. [5] applied the network science approach to evaluate vulnerable lines, stations, and sections of the metro network in Beijing, China.

Studies examining the impact of unplanned disruptions on passenger response are rather limited. Most studies focus on planned, long-term disruptions [6]. Furthermore, most of the unplanned disruption studies use traditional manual data collection methods, such as stated preference (SP) or revealed preference (RP) surveys. For example, Lin et al. [7] designed a joint RP and SP survey to study the pre-trip and en-route passengers' response to metro disruptions in Toronto, Canada. Using SP and RP data, Pnevmatikou et al. [8] developed a nested logit route choice model to study passengers' response to a 5-month metro line closure in Athens, Greece. The study revealed that passenger income and work flexibility significantly affected the observed mode shifts. Shires et al. [9] estimated demand impacts due to planned disruptions between London and Cardiff (Wales) using RP and stated intentions (SI) data. They found that bus bridging services covered only one third of the metro demand, and passengers showed little behavioral change during disruptions. Zhu et al. [10] used a panel survey to investigate planned metro disruptions for the Safe Track project in Washington, D.C. They found that the three most common responses to disruptions were not traveling, changing modes, or changing departure time. Currie and Muir [11] conducted a web survey among metro users in Melbourne, Australia. The findings reinforce the importance of information provision during disruptions, as $68 \%$ of respondents were willing to wait/use shuttle buses despite their low satisfaction with them. Li and Wang [12] developed an evacuation model to analyze evacuees' alternative options using an SP survey. The case study in Shanghai metro shows that besides bus bridging service, other alternative modes (e.g. taxi) are useful to mitigate disruption impacts.

Despite the fact that RP and SP surveys are widely used to understand passenger behavior, Sanko [13] reported that they have many limitations. For example, the SP surveys can be unreliable. The respondents may report differently from their actual behavior since the survey questions present hypothetical situations. SP surveys could also generate many non-usable values due to the lack of corroboration with other results and hypothetical bias [14]. In addition, survey data are limited in sample size, variations and treatment of variable correlations [15]. While surveys provide useful insights into general responses and perceptions, it is difficult to extract deep insights on disaggregated responses, especially the unplanned disruptions that vary in scale, location, and time.

With the increasing availability of AFC and automated vehicle location (AVL) data, a plethora of studies used these data to assess system performance and understand passenger behavior under typical conditions (i.e. no demand change or disruptions) [16-19]. The detailed information and insights on both system dynamics and passenger behavior can facilitate effective decision-making to improve transit services. However, studies using AFC and AVL data to explore the impact of unplanned disruptions on both system performance and individual response are limited. Sun et al. [20] developed a Bayesian method to identify the disruption. They used AFC data to evaluate the effect of an incident (unplanned disruption) in Beijing's railway network focusing on passengers' travel time and flows. Silva et al. [21] propose an approach to analyze large-scale massive transportation systems during unplanned disruptions. They estimated the disruption effects on passenger volumes during incidents using smart card data. Van der Hurk [22] developed a model based on smart cards to forecast the route choices of passengers impacted by disruptions under different scenarios. The study shows that operators can help passengers to minimize their overall inconvenience by providing individual advice.

These studies demonstrated that a data-driven approach is greatly beneficial to understand how the metro system impacts passengers' trips from various directions. However, they did not cover the analysis of passenger mobility, system performance, and other factors comprehensively. Some authors only focused on passengers' delays or volumes. Others concentrated on the analysis of mode choice or demand pattern. To get a deeper understanding of an unplanned incident's impacts on passengers, it is necessary to develop a systematic approach to investigate the incident from various perspectives using smart cart data. This paper proposes a data-driven approach, using AFC and AVL data, to systematically evaluate the impact of unplanned disruptions on both system performance and passenger 


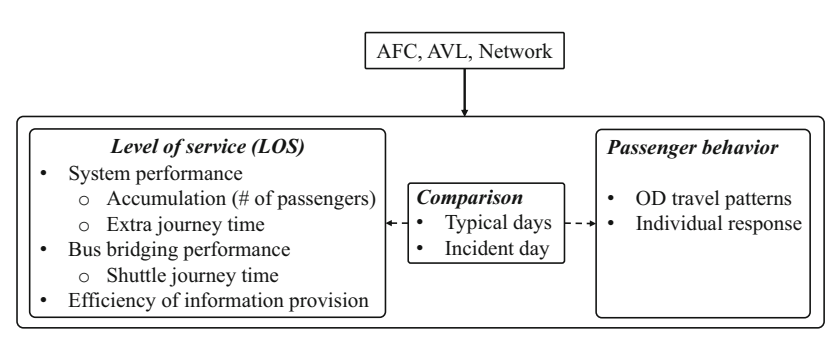

Fig. 1 Data-driven approach for disruption analysis

behavioral response in urban railway systems. A case study using data from a metro system is performed to illustrate the usefulness and value of the approach. The approach is general and could be applied to disruptions in transit systems that have closed AFC systems (recording both entry and exit transactions).

The rest of the paper is organized as follows: The Methodology section proposes the analysis framework and develops performance metrics to evaluate the impact of unplanned disruptions on the level of service and passenger behavior. The Case Study section illustrates the proposed approach and discusses empirical findings using a major incident in a busy metro system. The final section summarizes the main findings and discusses future directions.

\section{Methodology}

Figure 1 shows the proposed data-driven approach for unplanned disruption analysis. It consists of metrics organized around two important dimensions: level of service (LOS) and passenger behavior. The level of service analysis explores the impacts of disruptions on system performance, with respect to operations, bus bridging services, and information provision. The behavior analysis explores the impacts of disruptions on aggregate demand patterns and individual responses.

The LOS (system performance, bus bridging services, and information provision) and individual behavior response under unplanned disruptions are analyzed by comparing the performance metrics between disruption and typical days (baseline, no disruption).

\subsection{Baseline Measurement}

The baseline performance measurement is estimated based on typical days. A typical day is a day having normal operations with regular demand patterns and no disruption. A baseline performance metric (e.g. travel time) is defined as the average value of the metric over a number of typical days having similar characteristics with the disruption day (e.g. the same day of the week), $X=\frac{1}{n} \sum x_{i}$, where $n$ is the number of typical days and $x_{i}$ is the performance metric value on day $i$.

The baseline (habitual) passenger behavior is inferred based on a selected user panel. A panel is a group of passengers who consistently use the system for the period of interest (i.e. before and after an incident). By comparing their behavior on typical days and the incident day, it allows us to understand how disruption impacts their regular travel choices given their trip characteristics (e.g. being inside/outside the system when the incident happened).

\subsection{Disruption Impact on Level of Service (LOS)}

To assess the impacts on the level of service, several metrics are developed from three aspects: system, bus bridging strategy, and information provision.

- System performance metrics include the number of passengers in the system (accumulation) or on a train line (platforms and trains), the average journey time at the system or line level, extra journey time at the OD level, and the movement of trains in space (trajectories)

- Shuttle bus performance metrics include the average and median journey time

- Information provision efficiency is measured by quantifying the behavior of passengers at the affected stations, including the number of passengers who tap-in and tap-out at the same station and the time they spent in the affected stations

To assess the disruption impact, the performance metrics for the incident day are compared against the corresponding metrics on typical days.

\subsubsection{System Performance}

Passenger accumulation at the system level: Passenger accumulation in the system by the time of interest $T$ ( $i n$ tegral upper limit) is the total number of passengers in the system by that time (either on platforms or in trains). This metric is useful to assess the impact of disruptions on the system from passengers' perspective in terms of crowding. It also provides information about the recovery time when the service returns to normal.

It is calculated as the difference between the actual number of passengers who enter the system and the actual number of passengers who exit the system by time $T$ [23].

$N_{a c c}(T)=\int_{0}^{T}\left[N_{\text {ent }}(t)-N_{\text {ext }}(t)\right] d t$

where $N_{a c c}(T)$ is the number of passengers in the system by the time $T . N_{\text {ent }}(t)$ is the number of passengers entering the 
system at time $t . N_{\text {ext }}(t)$ is the number of passengers exiting the system at time $t$.

Passenger accumulation at the line level: Passenger accumulation at the line level is the total number of passengers on platforms and in trains of a service line. It is estimated as the difference between the number of passengers who enter or transfer into any station of the line and the total number of passengers who exit or transfer out of any station of the line.

$$
\begin{aligned}
N_{l-a c c}(T)= & \int_{0}^{T} X_{l-\text { in }}(t) d t-\int_{0}^{T} X_{l-\text { out }}(t) d t+\int_{0}^{T} Y_{l-\text { in }}(t) d t \\
& -\int_{0}^{T} Y_{l-\text { out }}(t) d t
\end{aligned}
$$

where $N_{l-a c c}(T)$ is the number of passengers accumulated on line $l$ at time $T . X_{l-i n}(t)$ is the number of passengers entering any station of line $l$ at time $t . X_{l-o u t}(t)$ is the number of passengers exiting any station of line $l$ at time $t$. $Y_{l-i n}(t)$ is the number of passengers transferring to line $l$ at time $t . Y_{l-o u t}(t)$ is the number of passengers transferring out of line $l$ at time $t$.

$X_{l-i n}(t)$ and $X_{l-o u t}(t)$ can be directly calculated from AFC records. The calculation of transferring passengers $Y_{l-i n}(t)$ and $Y_{l-o u t}(t)$ is not straightforward. We estimate the number of transfer passengers (in/out the line) at time $t$ by assigning OD flows to paths using appropriate path choice fractions (obtained from survey data [24]) and taking into consideration the journey time from the origin to the transfer stations of the line.

$$
\begin{array}{r}
Y_{l-\text { in }}(t)=\sum_{\text {od }} \sum_{p \in P_{\text {od }}} \sum_{s \in S_{l}} \pi_{\text {odh }-p} \cdot q_{\text {odh }} \cdot \delta_{s p-l-\text { in }} \\
Y_{l-\text { out }}(t)=\sum_{\text {od }} \sum_{p \in P_{\text {od }}} \sum_{s \in S_{l}} \pi_{\text {odh } p} \cdot q_{\text {odh }} \cdot \delta_{s p-l-\text { out }} \\
h=t-\tau_{\text {osp }}
\end{array}
$$

where $q_{\text {odh }}$ is the passenger flow of OD pair $(o, d)$ in time period $h . \pi_{o d h-p}$ is the fraction of passengers of OD pair $(o, d)$ using path $p \in P_{o d}$ at entry time period $h . \delta_{s p-l-i n}=1$ if station $s$ is the transfer station into line $l$ along path $p ; 0$ otherwise. $\delta_{s p-l-o u t}=1$ if station $s$ is the transfer station out of line $l$ along path $p ; 0$ otherwise. $\tau_{o s p}$ is the journey time from origin $o$ to station $s \in S_{l}$.

Average journey time: The system-level journey time is the average journey time of all trips in the system. The linelevel journey time is the average journey time of all trips on a service line. Journey time estimates delays and are used to assess how long it takes before journey time return to normal. Since journey time includes waiting and transfer time, the metric is also useful to characterize the severity of disruptions from the passengers' perspective. The metric is calculated by time period (15-minute intervals) using AFC data.

Extra journey time: OD-based analysis provides a more detailed understanding of how the incident impacts propagate through the network. In [25] the author introduces the concept of excess journey time (difference between the actual and a reference journey time, e.g. timetable). In this study we use an extra OD journey time, which is the difference between the median OD journey time on the incident day and on typical days. The metric is calculated by time period (15-minute intervals) using AFC data.

Train trajectories: From the operating aspect, the train frequency is useful to evaluate metro service performance. A comparison of the train trajectories on the incident day and on a typical day using AVL data provides useful insights into system performance.

\subsubsection{Bus Bridging Performance}

When disruptions happen, metro agencies often provide shuttle bus services to connect the disrupted stations. The shuttle service performance is assessed by comparing the average shuttle bus journey times metro service journey time for certain OD pairs. The shuttle bus journey time is approximated from AFC data of those users who tapped in and out at stations covered by the shuttle service and then tapped in at metro stations (also served by the shuttle service).

\subsubsection{Efficiency of Information Provision}

To evaluate the information efficiency, this analysis uses a select group of passengers who tap-in and out at the same station impacted by the disruption. The interpretation is that these passengers tapped-in at a station, found no service, and tapped-out at the same station. An increasing number of passengers with such behavior during a disruption may be an indicator of insufficient and/or ineffective information outside the station. The total number of passengers who tap-in and tap-out at the same station over all affected stations, and its ratio over the total number of tap-in passengers, are used as performance metrics to assess that. Furthermore, the time that passengers spent in the station before they tapped out at the same station is used to measure the effectiveness of information provision (inside the station). 


\subsection{Disruption Impact on Passenger Behavior}

\subsubsection{OD Travel Patterns}

Using OD passenger flow patterns, the analysis aims at understanding how passengers change their origins, destinations, and departure time during disruptions. The demand change is measured as the difference between the demand on a typical day and the incident day for the same OD pair and time period. The demand change over time provides a better understanding of how the impact of disruption propagates through the entire metro network.

\subsubsection{Individual Response}

Individual response to a disruption depends on many factors, including incident type and severity, origin and destination of passengers' trips, and whether the passenger was already in the system, etc.

Figure 2 shows the analysis framework of the individual response to a disruption. Passengers may belong to one of 4 groups depending on how their trips are impacted geographically (location). Passengers in each group are further categorized into 2 groups based on their trip timing (inside or outside the system) when the disruption occurs. Given their trip characteristics, passengers may change travel decisions (travel or not). If they travel, they may change the usual departure time, used OD stations and travel modes.

Spatial trip characteristics: Based on the spatial characteristics of their trips, relative to the location of the incident, passengers are classified into four groups.

- Group 1: Passengers whose origin stations are impacted by disruptions, but destinations are not.

- Group 2: Passengers whose destination stations are impacted by disruptions, but whose origins are not.

- Group 3: Passengers whose origin and destination stations are impacted by disruptions.

- Group 4: Passengers whose origin and destination stations are not impacted by disruptions, but their path goes through impacted stations.

Trip timing: When a disruption occurs, passengers may already be inside the system, or on their way to a station, or may not have even started their trip. Considering the trip timing, there are two subgroups: (a) passengers who are inside the system when the incident occurs; and (b) passengers outside the system. They may respond differently to the disruption. For example, passengers who are inside the system when a disruption happens, could either change destinations or tap-out from stations and switch to other modes. Passengers who have not yet departed (not yet tapin at stations) have more options, such as not travel, change departure time, change origin/destination stations, or switch to other modes.

Behavioral response: Depending on the spatial features and timing of their trips relative to the timing and location of the incident, the possible behavioral responses are as follows:

- Perform travel: Passengers decide to travel using the metro or avoid it (cancel trips or using non-metro methods) during the disruption period on the incident day

- Temporal change: Passengers who perform their planned trips may postpone their trips for a later time or undertake trips at the same time as planned during the disruption

- Spatial change: Passengers who perform trips may decide to change their typical origin or destination stations to be able to complete their trips during the disruption or keep using the same OD pairs as typical days

- Mode change: Passengers may perform their planned trips by utilizing the shuttle service, or keep using the metro during the disruption

Behavioral inference method: To infer individual response to a disruption, a panel of passengers is used. The passengers in the panel are those who consistently use the same OD pair and travel during similar time periods on typical days. Specifically, a panel is formed to include passengers who are impacted by the disruption and:

- Travel the same OD pairs on typical days

- Tap-in or tap-out on typical days during the disruption period on the incident day

Such a panel ensures that passengers are likely to travel on the incident day and are also impacted by the disruption. A panel-based analysis facilitates a more precise identification of spatiotemporal variations and inference of behavioral responses to the incident.

The behavior changes are inferred by comparing the panel members' responses on the incident day with their baseline behavior on typical days. The inference rules and process using AFC data are as follows:

1. Identify passengers who perform trips Those are the panel members who have at least one trip (i.e. AFC record) using the metro service after a disruption occurred on an incident day. Others either do not travel or avoid using the metro on that day.

2. Infer passengers who change travel time Out of panel members who perform trips on the incident day, those who depart later on that day than what they usually do on typical days (i.e. median departure time) are inferred as changing departure time. 
Fig. 2 Analysis framework of individual response

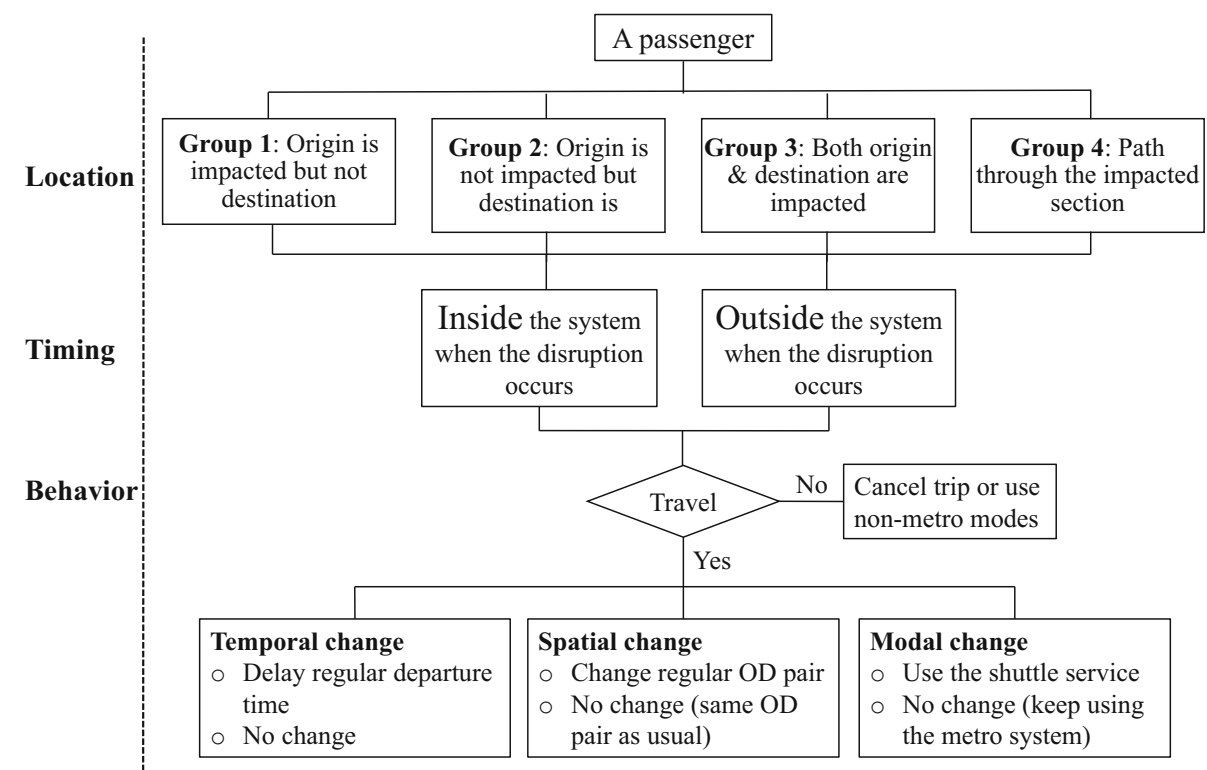

3. Infer passengers who change stations Out of panel members who perform trips on the incident day, those who used different transit stations on that day from what they usually use on typical days are inferred as changing stations.

4. Infer passengers who change modes Figure 3 describes the mode change inference process for panel members who perform trips on the incident day. The panel members who used the same OD pairs on the incident and typical days are inferred as "no mode" change. For panel members used different OD pairs, they are inferred as either "changed mode" or "not" depending on whether the changed stations are covered by the shuttle service or not.

Shuttle bus users are inferred as those who tapped in and tapped out from an impacted metro station and subsequently tapped-in at a station served by the shuttle service. It is assumed that they enter an impacted station first, experience delay and/or receive information about the incident. Subsequently, they tap-out from the original station and use the shuttle to connect to another station (not impacted) from which they continue their trips. The shuttle bus journey time is the difference between the tap-in time at the non-impacted station and tap-out time from the impacted (original) station.

\section{Case Study}

The AFC data provides detailed and accurate transaction information of individual trips, including the unique, anonymized smart card ID, tap-in station/timestamp, tapout station/timestamp, card type and fare. The AFC data in metro network is of high quality with less than $0.01 \%$ records missing field values (e.g., a trip transaction record with no tap-in or tap-out information). The transaction records with missing fields are not used in the study. The individual response to the disruption used a panel of users who are impacted by the disruption and travelled during the disruption time on typical days. Various responses are inferred by tracking their travel records after the disruption and comparing with these before the disruption and on typical days (Figs. 2 and 3).

The case study uses data from a major metro system with several million passenger trips per day. AFC and AVL data are used for the analysis. AFC data provide information about tap-in station/time and tap-out station/time for each passenger. AVL data includes information of train ID, departure/entry stations and time. A major incident is used to illustrate the proposed approach (Fig. 4). It occurred around 11:00 and trains were completely suspended until

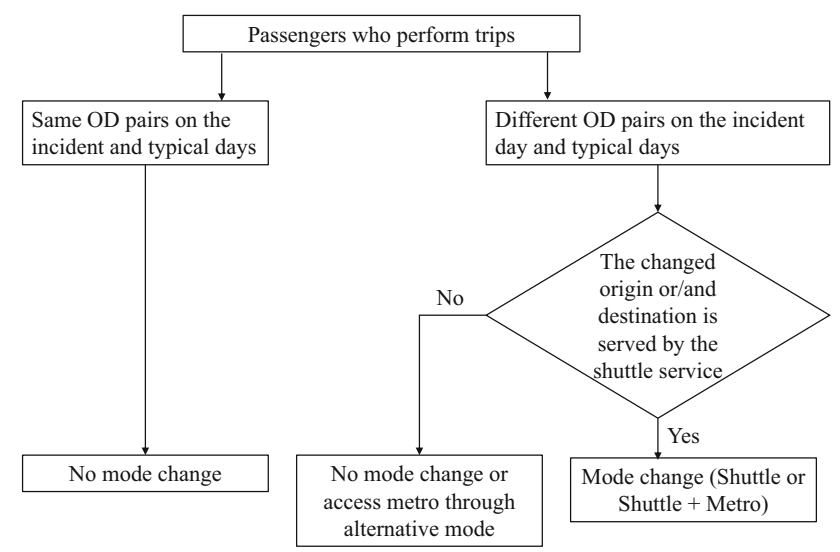

Fig. 3 Mode change inference process using AFC data 
11:38. The train service operated with increased headways until 15:29. Bus bridging services were provided between stations S6 and S1/S2 starting around 12:30 and ending at 16:00.

The baseline conditions are established using two similar typical days, representing the same day of the week as the incident day and in the same month. Figure 5 shows the passenger demand (total tap-ins), accumulation and average journey time (in 5-minute intervals) for the two typical days. It indicates that passengers use the system in a similar way, spending, on average, the same amount of time in the system. As Fig. 5 shows, the passenger demand, accumulation, and journey time are similar in these typical days across the entire day. The average value of these days (by time interval) is used as the baseline.

\subsection{Disruption Impact on the Level of Service}

\subsubsection{System Performance}

Figure 6 shows passenger accumulation at the system-level by time of day (in 5-minute intervals). The blue lines indicate the start and end time of the incident. The red line shows the passenger accumulation on the incident day while the black line shows the baseline (averaged over these two typical days). The passenger accumulation on the incident day is consistently higher during the disruption period and the trend continues until 17:00, though the incident ended at 15:29. The largest difference in accumulation happened around 13:30 (around 17,000 more passengers per 5 minutes are inside the system, $23 \%$ above the baseline).

Figure 7 shows passenger accumulation on the impacted line (in 5-minute intervals). The number of accumulated passengers started to increase when the incident started, and the impact continued until 16:30. Compared to the passenger accumulation at the system level, the disruption impact on the impacted train line is more significant. At its peak, it is about $65 \%$ higher than the baseline (additional 12,000 passengers per 5 minute). However, the duration of the impact propagates longer at the system level than the

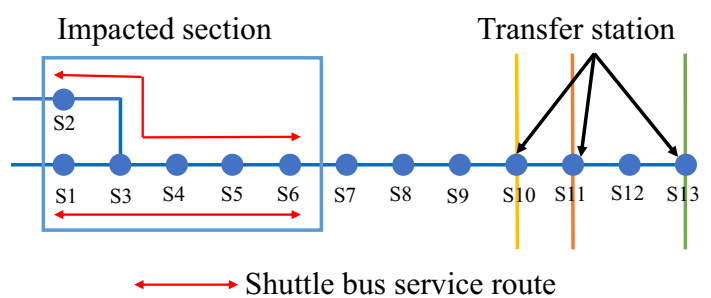

Fig. 4 Impacted line section and shuttle service routes

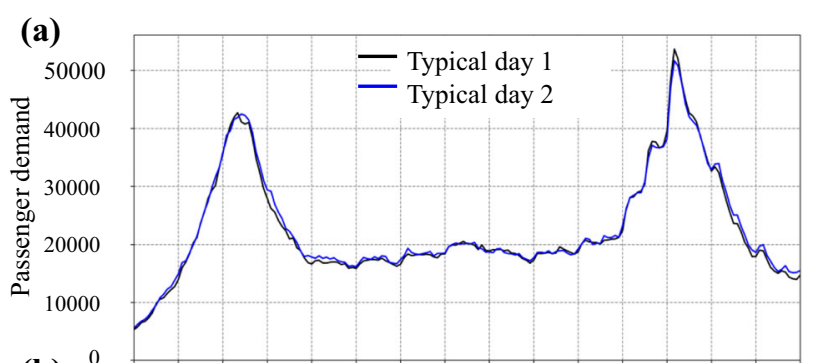

(b)

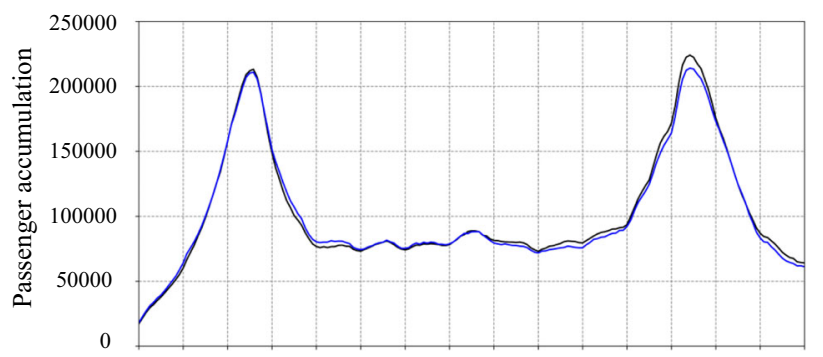

(c)

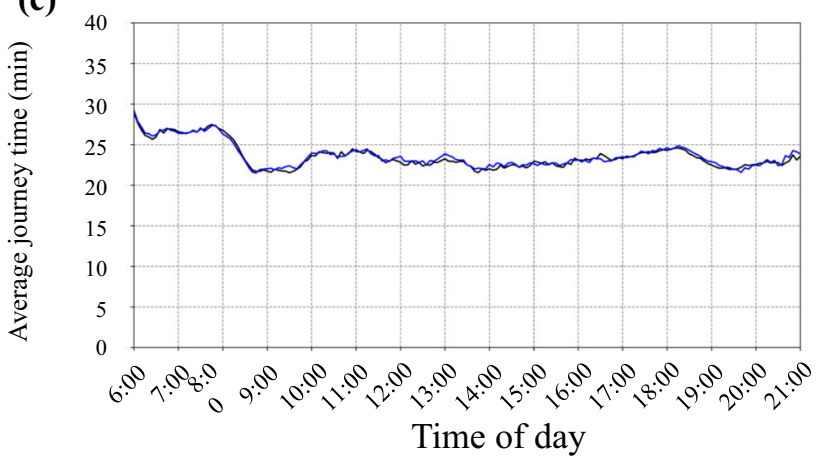

Fig. 5 Typical days. a passenger demand, b passenger accumulation, c average journey time

line level, and the recovery continues even after the metro service is fully restored.

Interestingly, the accumulation decreases around 11:30-11:45, then increases again. This corresponds to the time when the train service resumed (11:30), but with a much lower frequency than baseline. The train service capacity is far from enough to accommodate demand or mitigate overcrowding under disruptions. It indicates that, besides fast service recovery, other mitigation strategies could also be important to better manage the demand, such as real-time service updates and travel recommendations, and the shuttle service.

Figure 8 shows the average journey time at the system level (in 5-minute intervals). The average journey time started to increase almost one hour before the incident occurred. At its peak (10:50-11:00), the average journey time increased by 3.5 minutes (13\% increase from baseline). This reflects the fact that some passengers, who boarded a train before the incident, are impacted by the incident in the middle of their journey. In addition, since 

at the system level
Fig. 6 Passenger accumulation
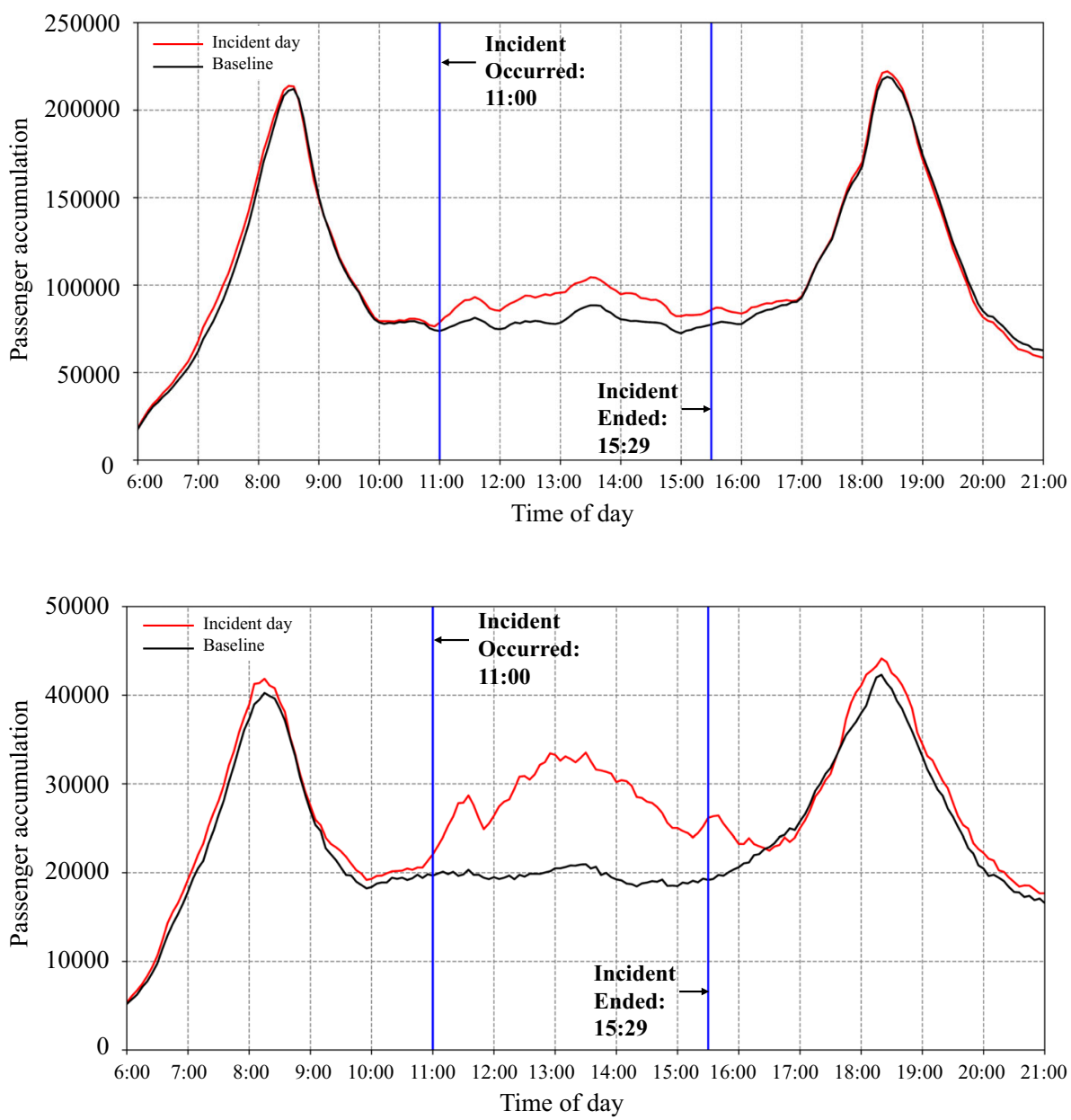

Fig. 7 Passenger accumulation on the impacted line

Fig. 8 Average journey time in the system

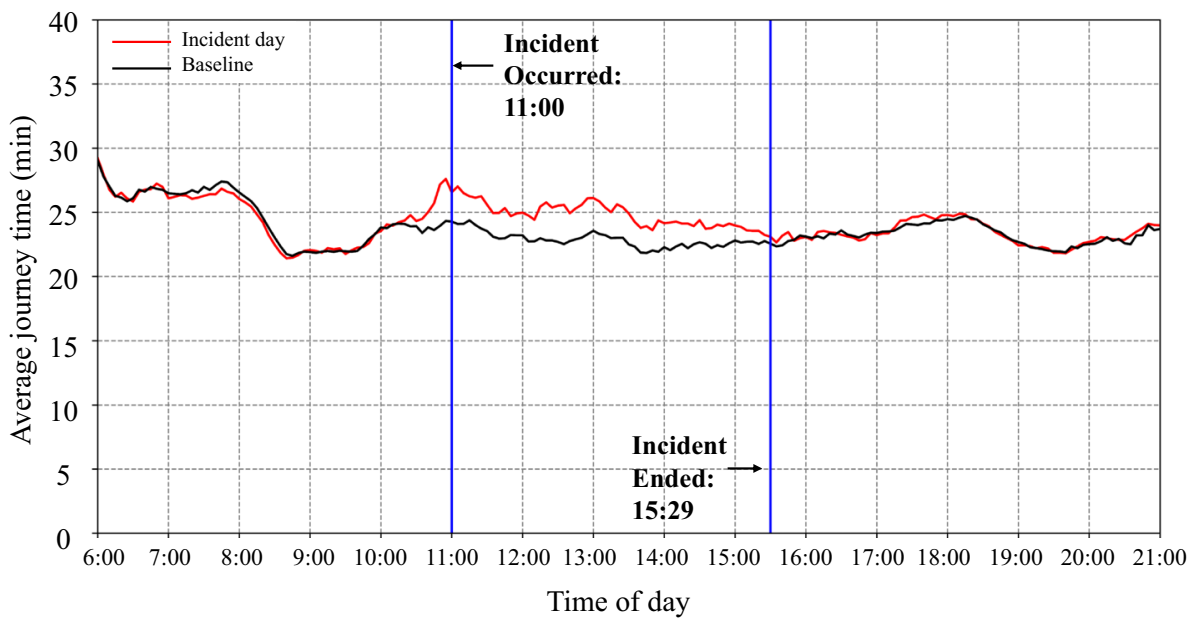

these passengers were inside the system when the incident happened, they experienced the impacts the most, possibly due to limited alternative options and travel information. As expected, the journey time is consistently higher than baseline during the incident time period (11:00-15:30) given the low-frequency train services. It recovers to the baseline around the incident end time.
Figure 9 shows the average journey time for all trips on or passing through the line impacted by the incident. As expected, passengers using the impacted line are impacted more severely by the disruption. Passengers who tapped-in at the impacted line when the incident happened, experienced an additional 21 minutes of journey time $(90 \%$ increase from baseline). Passengers who tapped-in about 
Fig. 9 Average journey time on the impacted line

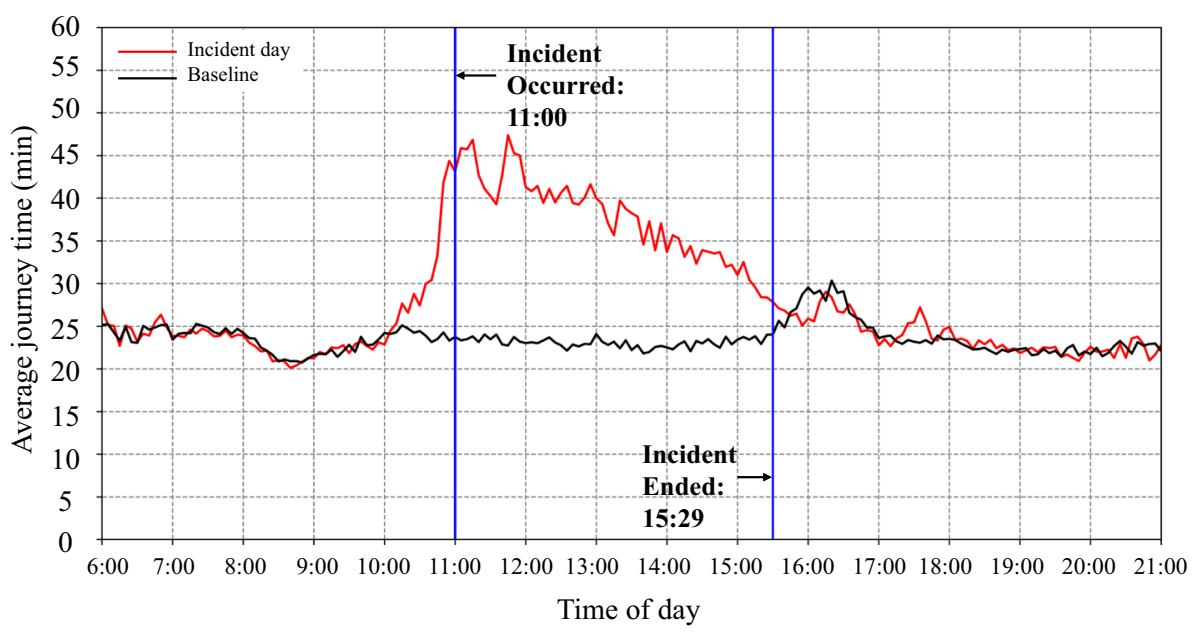

11:30 experienced a smaller decrease in journey time than those who tapped-in around 11:00. This corresponds to the time when the train service started to operate (less frequently than normal).

The proposed metrics of passenger accumulation and average journey time, either at the system or line-level, capture the impact of disruptions on passengers. Even if the service is restored, the impact on passenger journeys lasts longer. Therefore, both metrics can be used to better estimate the actual recovery time of an incident (in addition to its duration), especially from a passenger's point of view.

AFC data allows the evaluation of the impacts at a higher resolution. Figure 10 shows the heatmap of extra journey time for all OD pairs over time. The extra journey time analysis is based on the passengers who use the metro service on the incident day using their tap-in and tap-out information. Extra journey time is defined as the difference in journey time between the incident and typical days (averaged in the one-hour interval). Each cell represents the extra journey time of an OD pair. The stations are arranged according to different train lines, separated by black lines. The impacted lines are indicated using the red arrows on the top-left corner.

As expected, passengers experience longer delays if they travel through the impacted section of the line. Between 12:00 and 13:00, it may take up to almost 83 minutes additional time from a station on a non-impacted line to the impacted station S1. Passengers entering the system at a station of a non-impacted line and transfer to the impacted line, may not be aware of the severity of the disruption, hence continue their trips, experiencing significant delays.

Interestingly, passengers who travel along the impacted section in the first hour after the incident do not always experience the worst delays. In this case, passengers who tapped-in between 12:00 and 13:00 experienced more delays than those who tapped-in between 11:00 and 12:00 (even though there was no train service for 30 minutes). This could be caused by the surge of passenger accumulation between 12:00 and 13:00 (Fig. 7), leading to denied boarding and long waiting on platforms. It also indicates that many passengers keep using the train service despite its long travel time, and they are reluctant to switch to choose other alternatives.
Fig. 10 Extra OD journey time in minutes: a tap-in between 11:00 and 12:00 (one hour after the incident); $\mathbf{b}$ tap-in between 12:00 and 13:00 (two hours after the incident)

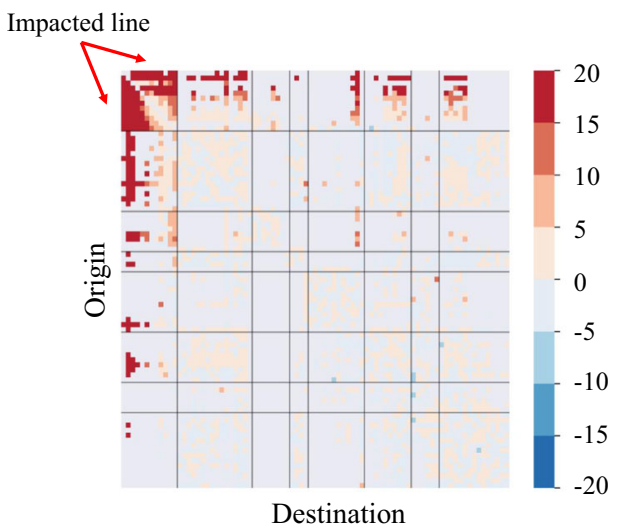

(a)

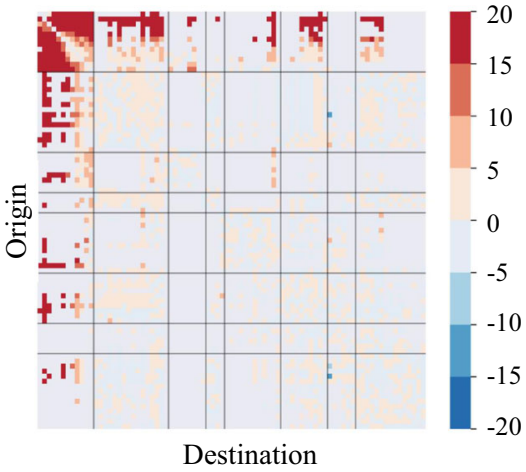

(b) 
Fig. 11 Train movement from stations $\mathrm{S} 13$ to S1/S2:

a Baseline day; b Incident day
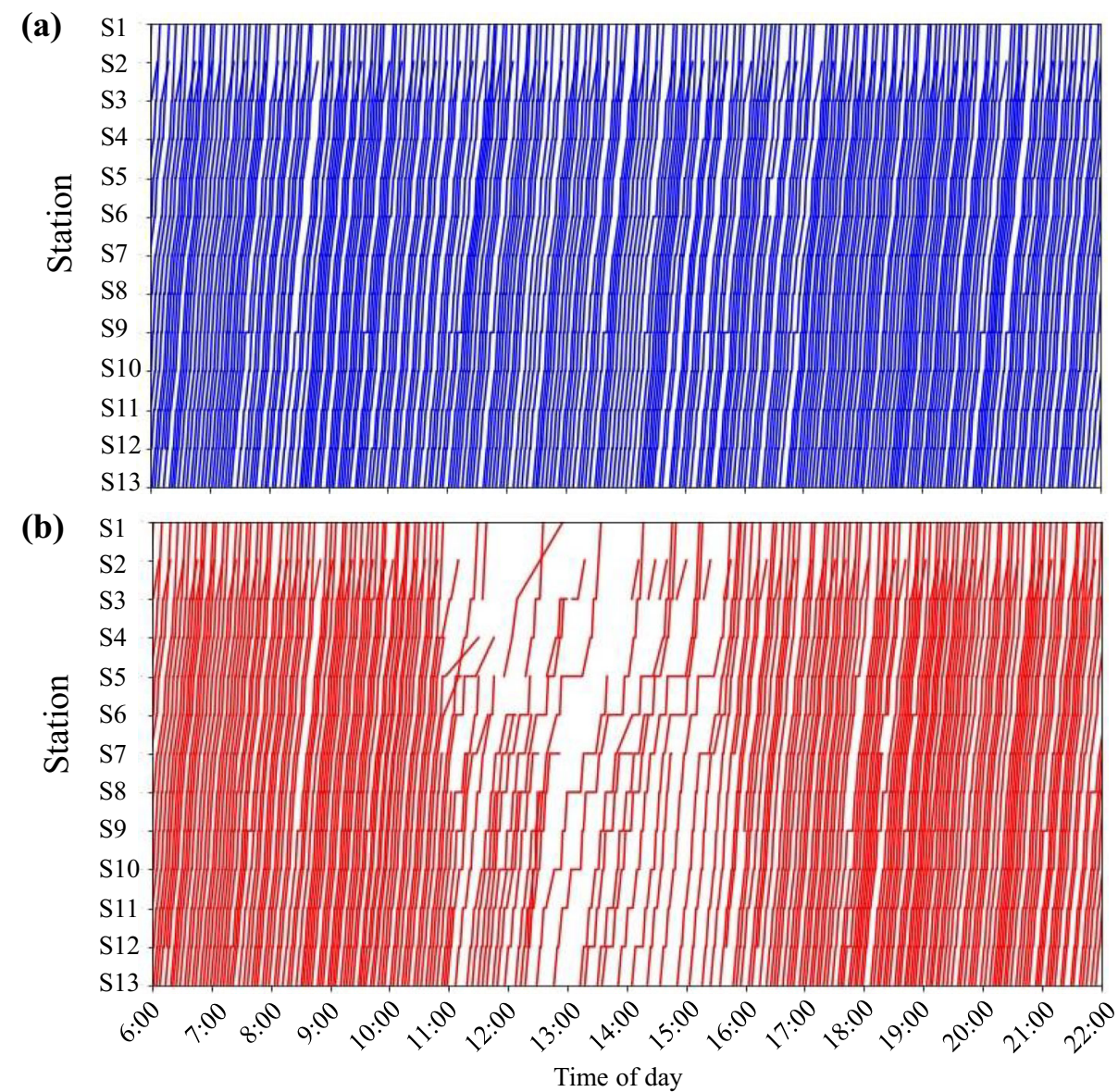

Figure 11 shows train movements on the impacted line on the incident day and on the baseline day from station $\mathrm{S} 13$ to $\mathrm{S} 1$ or $\mathrm{S} 2$. The railway line starts at station 13 and branches at station S3 to terminate at station S1 or S2. During the disruption period fewer trains operated than in the same period on a typical day. Especially, the service

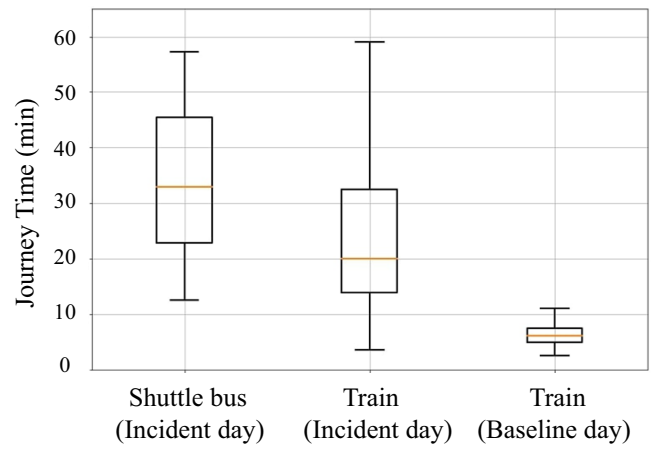

Fig. 12 Journey time from S3 to S4 by shuttle and train (incident and typical day) during the disruption period. frequency is significantly lower between stations S5 and S1 or S2.

\subsubsection{Bus Bridging Performance}

Figure 12 compares the journey time experienced by passengers using the shuttle and train services during the incident, and the journey time of metro users on a typical day. The shuttle bus journey time is estimated using the shuttle bus users identified from the AFC data who tap-in and tap-out at an impacted station and then tap-in at a station served by the provided shuttle service. It shows that shuttle bus users experienced longer but comparable journey time than metro users, probably caused by longer searching and queue waiting time or traffic on roads.

In general, using shuttle services is a reasonable means to mitigate the impacts of an incident. Besides the importance of the timely deployment of the shuttle services, upto-date information to passengers regarding both the status of the subway service and shuttle services is also critical. Such information can be disseminated through a variety of communication channels e.g. mobile phones, platform displays, and announcements. 


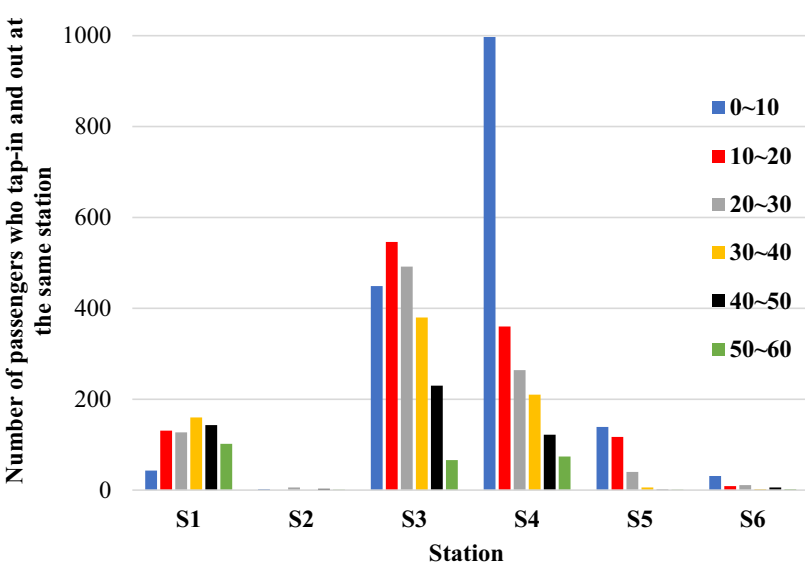

Fig. 13 Number of passengers who tap-in and out at the same station and distribution of the time spent in the station on the incident day

\subsubsection{Efficiency of Information Provision}

Regardless of disruptions, passengers are still able to enter the impacted stations. AFC data also indicates that passengers may tap-in and tap-out at the same station. This behavior can be used as an indicator of information effectiveness during disruptions. The time passengers spent inside the station before they leave the station to pursue other options is a useful metric of information efficiency. It can be calculated from their tap-in and tap-out time at the same station.

Figure 13 shows the number of passengers who tap-in and out at the same station and the distribution of the time spent in the station on the incident day. The number of passengers who tap-in and out at the same impacted station varies by station. For example, 2163 passengers tapped in and out at station S4, while only 12 passengers behaved in this way at station S2. Also, the distribution of the time spent in the station varies by station. For example, most passengers spent less than 10 minutes at station S4 and 10-30 minutes at station S3. Possible reasons can include
Table 1 Behavioral response of passengers who are outside the system when the incident occurred

\begin{tabular}{lccc}
\hline Response & Group & Group & Group \\
& $1(\%)$ & $2(\%)$ & $3(\%)$ \\
\hline Perform travel & 60.5 & 52.9 & 52.5 \\
Spatial change & 49.7 & 33.7 & 50.9 \\
Temporal change & 2.4 & 6.0 & 7.9 \\
Mode change & 29.1 & 10.8 & 36.0 \\
\hline
\end{tabular}

communication efficiency, alternative travel options, and operation capacity at the impacted stations.

\subsection{Disruption Impacts on Passenger Behavior}

\subsection{OD Travel patterns}

Figure 14 shows the heatmap of demand changes for all OD pairs after the incident happened. The demand change is the difference in passenger demand between the incident day and the baseline (by the hour).

The entry demand of directly impacted OD pairs decreased mainly during the first two hours of the disruption. Demand for other OD pairs may have changed due to the redistribution of passenger flows, e.g. passengers traveling using other modes and other metro stations to continue their trips. The largest passenger flow decrease happened for OD pair S3-S2 between 11:00 and 12:00, accounting for a $45 \%$ decrease from a typical day. At the same time period, the passenger flow of OD pair S2-S3 increased by $28 \%$, compared to baseline. Between 12:00 and 13:00, the largest passenger flow decrease happened for OD pair S3-S4, a 72\% decrease from the baseline. At the same time period, the passenger of OD pair S1-S3 increased by $130 \%$.

Compared with the extra journey time in Fig. 10, the disruption impact on OD passenger demand does not propagate as much at the system level, confined mainly at
Fig. 14 Passenger demand changes: a tap-in between 11:00 and 12:00 (one hour after the incident); b tap-in between 12:00 and 13:00 (two hours after the incident)

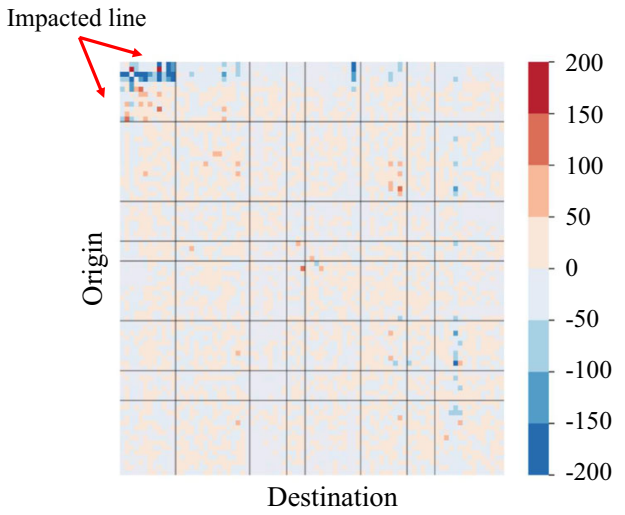

(a)

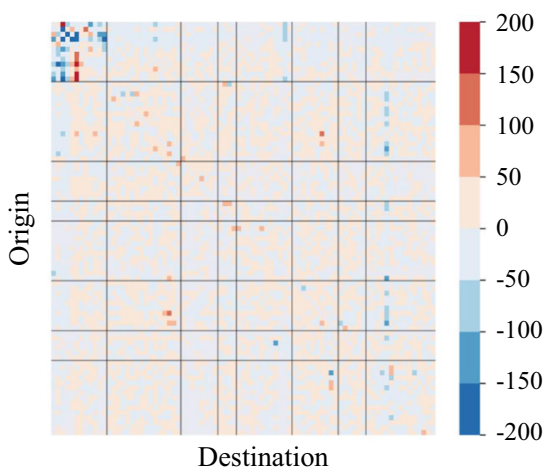

(b) 
Table 2 Behavioral response of passengers who are in the system when the incident occurred

\begin{tabular}{llll}
\hline Response & $\begin{array}{l}\text { Group } \\
1(\%)\end{array}$ & $\begin{array}{l}\text { Group } \\
2(\%)\end{array}$ & $\begin{array}{l}\text { Group } \\
3(\%)\end{array}$ \\
\hline Perform travel & 100 & 100 & 100 \\
Spatial change & 9.0 & 26.1 & 28.6 \\
Temporal change & 0.0 & 0.0 & 0.0 \\
Mode change & 1.6 & 17.4 & 19.0 \\
\hline
\end{tabular}

disrupted stations. Passengers do not necessarily avoid traveling using the metro during the disruption. This suggests that mitigation strategies to prevent overcrowding on train cars or platforms are also important.

For some OD pairs (S2 to S3 and S1 to S3), passenger demand increased significantly during the disruption while other OD pairs (S3 to S2 and S3 to S4) decreased. This may be because many passengers who planned to enter at station S3 switched to station S2 or S1 using the shuttle or other modes during the disruption period. Other reasons could be that passengers using station S2 and/or S1 have a strong preference for the metro system, or they did not receive sufficient information regarding the status of train operations.

\subsubsection{Individual Response}

To understand the individual response during the disruption, a panel of 2035 users who travel the same OD pairs, and travel during the disruption (incident day) time period in typical days is used. Table 1 shows the behavioral response of different passenger groups (Fig. 2) under the disruption. These passengers were outside the system when the incident occurred. Note that passengers in group 4 are those who go through impacted stations, but their OD pairs are not impacted. There are no such passengers in the panel, as the impacted section is at the line's terminals.

- From the passengers whose typical origins, but not destinations, were impacted (Group 1), 60\% performed trip travels, and $40 \%$ either completed trips without using the metro or did not travel during the disruption period. Among those who performed trips, 50\% changed origins or destinations or both, but $98 \%$ kept their original departure time.

- Compared to Group1, passengers whose typical destinations, but not origins, were impacted, Group 2 were less likely to travel, change stations or use the shuttle, but more likely to change departure time, since they have more flexibility in making travel choices at origins (use alternative routes or destination stations).

- Passengers in Group 3 whose typical origins and destinations were both impacted wee most likely to change departure time, stations and also use shuttle buses if they travel, since they need to plan for both ends of their trips.

Table 2 summarizes how passengers who were in the system when the incident occurred responded to the disruption. As they are already in the system, they have no choice over whether or not to travel or to change their departure time. Passengers in Groups 2 and 3 have similar behavioral responses, and they are more likely to change stations and use the shuttle service compared to those in Group 1. That could be because passengers whose typical origins are impacted are prone to wait for the train service since they are already in the system or forced to wait if there are no alternative options at the impacted origins. Also, Group 1 passengers may have little intention to change destinations since their destinations are not impacted.

In summary, over $50 \%$ of passengers switch to alternative modes to avoid using the metro if they are outside the system when the disruption happens. Once passengers decide to use the system, few change their typical departure time, although many of them change either origins or destinations or both. On the other hand, among the passengers who are inside the system when the disruption happens, few actively change their original destinations or wait for the shuttle service to continue their trips. This may indicate that information about the shuttle service or alternative mode options needs to be effectively and immediately provided after the disruption happens, so passengers would be able to leave from the metro station.

\section{Conclusion}

The paper proposes a data-driven approach using AFC data to analyze unplanned disruption impacts on metro performance and passenger behavioral responses. The approach provides insights to better understand the impacts of disruptions on the level of service (system, shuttle bus, and information) and possible passenger behavior to improve existing disruption management strategies. The proposed methodology can be applied to metro systems with closed fare systems (both tap-in and tap-out transactions).

The proposed metrics and inference methods using AFC data are useful for understanding how passengers adjust their trips and use the shuttle service based on their location and time. From a transit agency point of view, the results indicate that it could be beneficial to provide timely and relevant information. Given the detailed nature of the AFC data, it is even possible that information can be personalized based on passengers' typical travel patterns. Bus 
bridging strategies can also be designed to better match passenger ODs.

The empirical analysis shows that the disruption impacts are network-wide. Furthermore, the impacts on passengers continue for a significant amount of time after the incident ended. The information provided in the first 30 60 minutes after a serious disruption is critical, as many passengers could wait for a long time inside the impacted station before leaving for alternative travel options. The behavior highlights the importance of real-time information and the need for that information to be disseminated efficiently. In this specific case study, more passengers prefer to use the metro, even if services are degraded, than taking the shuttle. A possible reason may be that shuttle-service journey time is longer than if the passengers had remained in the system. Shuttle users spent more than 1.5 times as much travel time as metro users during the disruption period (despite the increased train headways and other delays).

The proposed approach is limited to metro systems with a closed fare system. However, the overall framework is useful for open systems as well if destination can be inferred for a significant fraction of the trips (e.g. Gordon et al. [26]). The method may not be applicable or may bias the results in situations where a significant amount of transaction records of some OD pairs is unavailable under severe disruptions (e.g., infrastructure failure or no ticket validation). The empirical findings are based on several disruption cases in the studied metro system, and caution is advised when extending the findings to other systems. Future research can focus on the design of effective shuttle services, considering passenger OD flows. Design of information provision can also have important impacts on passenger experience during an incident.

Acknowledgments The authors thank the transit agency for their support and data for this research.

\begin{abstract}
Author's contributions The authors confirm contribution to the paper as follows: study conception and model design: TL, ZM, HNK; data collection and implementation: TL, ZM; analysis and interpretation of results: TL, ZM, HNK; draft manuscript preparation: TL, $\mathrm{ZM}$, HNK. All authors reviewed the results and approved the final version of the manuscript.
\end{abstract}

Funding The study was partially funded by a major transit agency in Asia.

Code availability Codes are available upon request.

\section{Declarations}

Conflict of interest The authors declare that they have no conflicts of interest.
Availability of data and material Data will be made available upon request.

Open Access This article is licensed under a Creative Commons Attribution 4.0 International License, which permits use, sharing, adaptation, distribution and reproduction in any medium or format, as long as you give appropriate credit to the original author(s) and the source, provide a link to the Creative Commons licence, and indicate if changes were made. The images or other third party material in this article are included in the article's Creative Commons licence, unless indicated otherwise in a credit line to the material. If material is not included in the article's Creative Commons licence and your intended use is not permitted by statutory regulation or exceeds the permitted use, you will need to obtain permission directly from the copyright holder. To view a copy of this licence, visit http://creativecommons. org/licenses/by/4.0/.

\section{References}

1. Dong H, Ning B, Sun X et al (2013) Emergency management of urban rail transportation based on parallel systems. IEEE Trans Intell Transp Syst 14(2):627-636. https://doi.org/10.1109/TITS. 2012.2228260

2. Jenelius E, Cats O (2015) The value of new public transport links for network robustness and redundancy transportmetrica a: transport. Science 11(9):819-835. https://doi.org/10.1080/ 232499(35),pp.1087232,2015

3. Adnan M, Pereira F C, Azevedo A L, Basak K, Koh K, Loganathan H, Peng Z H, Ben-Akiva A (2007) Evaluating disruption management strategies in rail transit using SimMobility Mid-term simulator: A case of Singapore MRT North-East line. Transportation research board 96th annual meeting 17-06587

4. Yap M.D, Cats O (2019) Analysis and Prediction of Disruptions in Metro Networks. 2019 6th International Conference on Models and Technologies for Intelligent Transportation Systems (MTITS), Cracow, Poland, pp 1-7. https://doi.org/10.1109/MTITS. 2019.8883320

5. Yin H, Han B, Li D, Wang Y (2016) Evaluating disruption in rail transit network: a case study of Beijing subway. Sci Direst. Procedia Eng 137:49-58

6. J Balog A Boyd J Caton (2003) The public transportation system security and emergency preparedness planning guide Volpe National Transportation Systems Center, Cambridge, Massachusetts, USA U.S., Department of Transportation Research and Special Programs Administration John A

7. Lin T, Shalaby A, Miller E (2016) Transit User Behavior in Response to Subway Service Disruption. Resilient infrastructure, CSCE Annual Conference, London, UK

8. Pnevmatikou AM, Karlaftis MG, Kepaptsoglou K (2015) Metro service disruptions: how do people choose to travel? Transportation 42:933-949. https://doi.org/10.1007/s11116-015-96564

9. Shires JD, Cabral MO, Wardman M (2019) The impact of planned disruptions on rail passenger demand. Transportation 46(1807-1837):4

10. Zhu S, Masud H, Xiong C, Yang Z, Pan Y, Zhang L (2017) Record. Journal of the, D.C, Transportation Research Record. J Trans Res Board 2649:79-88

11. Currie G, Muir C (2017) Understanding passenger perceptions and behaviors during unplanned rail disruptions. Sci Direct, Trans Res Procedia 25:4392-4402. https://doi.org/10.1016/j.trpro.2017. 05.322 
12. Li J, Wang X (2020) Multimodal evacuation after subway breakdown: a modeling framework and mode choice behavior. Trans Res Interdiscip Perspect 6:100177

13. Sanko N (2001) Guidelines for Stated Preference Experiment Design in Champs-sur-Marne, France. Master dissertation, Ecole Nationale des Ponts et Chaussees

14. McFaddden D (2017) Chapter 6 - Stated preference methods and their applicability to environmental use and non-use. Contingent Valuation of Environmental Goods, pp 153-187. https://doi.org/ $10.4337 / 9781786434692.00012$

15. Kroes EP, Sheldon RJ (1988) Stated preference methods: an introduction. JTEP 22(1):11-25

16. Welch TF, Widita (2019) Big data in public transportation a review of sources and methods. Transp Rev 39(6):795-818. https://doi.org/10.1080/01441647.2019.1616849

17. Koutsopoulos H N, Ma Z, Noursalehi P, Zhu Y (2019) Chapter 10 - Transit Data Analytics for Planning, Monitoring, Control, and Information. In Mobility Patterns, Big Data and Transport Analytics: Tools and applications for modelling, pp 229-261. https:// doi.org/10.1016/B978-0-128129 70-8.00010-5

18. Lepage S, Morency C (2019) Impact of atypical events on transportation demand, 5th international workshop and symposium, TRANSITDATA2019. France, Paris

19. Ghofrani F, He Q, Goverde RMP, Liu X (2018) Recent application of big data analytics in railway transportation system: a Survey. Trans Res Part C Emerg Technol 90:226-246
20. Sun H, Wu J, W Yan Gao LXZ (2016) Estimating the influence of common disruptions on urban rail transit networks. Trans Res Part A Policy Pract 94:62-75

21. Silva R, Kang SM, Airoldi EM (2015) Predicting traffic volumes and estimating the effects of shocks in massive transportation systems. Proc Natl Acad Sci 112(18):5643-5648. https://doi.org/ 10.1073/pnas. 1412908112

22. Van der Hurk E (2015) Passengers, Information, and Disruptions, $\mathrm{PhD}$ dissertation, Erasmus University Rotterdam, Rotterdam, Netherlands

23. Freemark Y (2013) Assessing journey time impacts of disruptions on London's Piccadilly line, Master dissertation, Massachusetts Institute of Technology, Cambridge, MA, US

24. Mo B, Ma Z, Koutsopoulos HN (2020) Capacity-constrained network performance model for urban rail systems. Trans Res Record: J Trans Res Board 2674(5):59-69. https://doi.org/10. 1177/0361198120914309

25. Zhao J, Frumin M, Wilson N, Zhao Z (2013) Unified estimator for excess journey time under before heterogeneous passenger incidence behavior using smartcard data. Trans Res Part C: Emerg Technol 34:70-88

26. Gordon J, Koutsopoulos H, Wilson N, Attanucci J (2013) Automated inference of linked transit journeys in London using faretransaction and vehicle location data. Trans Res Record: J Trans Res Board 2343:17-24. https://doi.org/10.3141/2343-03 